\title{
Demographic and health factors associated with pandemic anxiety in the context of COVID- I 9
}

\author{
Eoin McElroy*I (D), Praveetha Patalay ${ }^{2}$ (D), Bettina Moltrecht ${ }^{3}$ (D), \\ Mark Shevlin ${ }^{4}$ (D) Adrienne Shum ${ }^{5}$ (D), Cathy Creswell ${ }^{5}$ (D) and \\ Polly Waite ${ }^{5}$
}

'Department of Neuroscience, Psychology and Behaviour, University of Leicester, UK

${ }^{2}$ Centre for Longitudinal Studies and Medical Research Council Unit for Lifelong Health and Ageing, University College London, UK

${ }^{3}$ Evidence Based Practice Unit, University College London and Anna Freud National Centre for Children and Families, London, UK

${ }^{4}$ School of Psychology, Ulster University, Coleraine, UK

${ }^{5}$ Departments of Experimental Psychology and Psychiatry, University of Oxford, UK

Objectives. The mental health consequences of COVID-19 are predicted to have a disproportionate impact on certain groups. We aimed to develop a brief measure, the Pandemic Anxiety Scale, to capture the specific aspects of the pandemic that are provoking anxiety, and explore how these vary by health and demographic factors.

Design. Data were from a convenience sample of parents $(N=4,793)$ and adolescents $(N=698)$ recruited in the first 6 weeks of lockdown.

Methods. Factor analytic and IRT methods were used to validate the new measure in both parent and adolescent samples. Associations between scores on the new measure and age, gender, household income, and physical health status were explored using structural equation modelling (SEM).

Results. Two factors were identified in both samples: disease anxiety (e.g., catching, transmitting the virus) and consequence anxiety (e.g., impact on economic prospects); and unique associations with health and demographic factors were observed.

Conclusions. Anxieties due to the COVID-19 are multifaceted, and the PAS is a short, reliable, and valid measure of these concerns. These anxieties are differentially associated with demographic, social, and health factors, which should be considered when developing strategies to mitigate the mental health impact of the pandemic.

This is an open access article under the terms of the Creative Commons Attribution License, which permits use, distribution and reproduction in any medium, provided the original work is properly cited.

*Correspondence should be addressed to Eoin McElroy, Department of Neuroscience, Psychology and Behaviour, University of Leicester, University Road, Leicester, LEI 7RH, UK (email: em447@leicester.ac.uk).

Joint senior author. 


\section{Statement of contribution}

\section{What is already known on this subject?}

- Evidence from previous pandemics and current nationally representative studies suggests that COVID- 19 is having a pronounced negative impact on public mental health.

- The majority of research in this area has focussed on average levels of mental distress in comparison with pre-pandemic norms.

- Little is known about the specific aspects of the pandemic that are causing worry/distress and whether there are meaningful group differences in the worries experienced.

\section{What does this study add?}

- The present study adds to the emerging COVID-19 literature, by demonstrating, via a newly developed measure of pandemic-related worry, that there are meaningfully distinct forms of anxiety emerging due to COVID-19, and that both demographic and health factors predict who experiences these worries.

- The study is also unique in that it includes a large sample of both adults and adolescents from an ongoing study into the mental health outcomes of COVID-19.

\section{Background}

The COVID-19 pandemic is arguably the largest public health crisis of the past century. In addition to the rising number of premature deaths and the burden placed on health services, COVID-19 also poses a significant challenge for population-level mental health (Holmes et al., 2020). Evidence from previous pandemics (Wu et al., 2009) suggests a pronounced negative impact on public mental health, and nationally representative panel studies in the United Kingdom have reported increase in mental health problems in the first 2 months of lockdown (Banks \& Xu, 2020; Shevlin et al., 2020). Moreover, the mental health consequences of a global pandemic are likely attributable to more than just illness and bereavement from the disease itself (Holmes et al., 2020). Due to the ongoing public health measures, the public are experiencing unprecedented levels of social distancing and isolation. Economic and educational disruption has left many people out of work, with increased responsibilities (e.g., home education and full-time work), and short-term financial difficulties. Beyond this, the economic repercussions of the pandemic are projected to be vast (McKibbin \& Fernando, 2020).

Although there are several ongoing investigations into the mental health of the population during the pandemic, the majority are concerned with levels of psychological distress in comparison with pre-pandemic norms (Banks \& Xu, 2020; Shevlin et al., 2020). Moreover, while several psychometric scales have recently been developed to assess COVID-19-specific anxiety, the majority treat such anxiety as a unique clinical entity, with the focus on symptoms (Ahorsu et al., 2020; Lee, 2020). To our knowledge, only one scale thus far has focussed on the specific aspects/ contextual factors of the pandemic that are provoking anxiety, the COVID Stress Scales (Taylor et al., 2020). This 33-item measure measures five dimensions of COVID19-specific anxiety (danger and contamination, socio-economic consequences, xenophobia, traumatic stress, and compulsive checking). The length of this measure, however, may preclude its use in large, population-based studies which are key in understanding the mental health burden of the pandemic. In the present study, we developed the Pandemic Anxiety Scale (PAS) to fill this need. 
The PAS is a brief 7-item measure that captures worries about the disease itself (e.g., catching, transmitting) and also short- and long-term outcomes associated with public health response (e.g., current income, economic prospects) and has been designed to be used with both young people and adults. This paper presents its initial psychometric investigation including factor structure, item distributions, reliability, and construct validity in a large sample of adolescents and adults in the United Kingdom.

Given that the COVID-19 pandemic has direct and indirect consequences that are more severe for certain groups (Office for National Statistics, 2020), our second aim was to explore the impact of demographic and health factors on pandemic-specific anxieties. For instance, most hospitalizations and deaths from the disease occur in those aged above 60 and with underlying health conditions (Jordan, Adab, \& Cheng, 2020). Conversely, young people and those with limited financial resources are particularly affected by the public health response to the outbreak (e.g., disruption to education and employment), with a looming recession threatening their economic and labour market prospects in the future (Ahmed, Ahmed, Pissarides, \& Stiglitz, 2020; Wang, Zhang, Zhao, Zhang, \& Jiang, 2020). By furthering our understanding of the types of worry experienced by the public, and how such anxieties vary by the above demographic and health factors, we may gain a better understanding of what aspects of the current situation are causing worry and in whom. Given the exploratory nature of this study and our PAS measure, we make no hypotheses about the strength or direction of these associations a priori.

\section{Methods}

\section{Participants}

Participants were from a UK-based longitudinal study investigating the mental health and well-being of parents/carers and children (aged 4-16 years) during the COVID-19 lockdown (Co-Space Study: Supporting Parents, Adolescents and Children during Epidemics). The aim of this survey was to track the mental health of children and young people throughout the COVID-19 pandemic in order to identify the parental practices that protect children from deteriorating mental health, and how such practices vary according to child and family characteristics. An opportunity sample of parents/carers were recruited, with the study advertised widely through various media formats and networks. The survey was completed online via Qualtrics. Following the parent-report section, an adolescent-report sub-survey was presented for children aged between 11 and 16 years (one child per family). Informed consent was sought from the parents for their portion of the survey, and from both parents and adolescents for the sub-study. Parents $(N=5,683)$ and adolescents $(N=749)$ completed a baseline survey between the 30th of March and the 29th of April 2020 (between 1 and 6 weeks into lockdown in the United Kingdom). Ethical approval for the study was granted by the central university research ethics committee at the $<$ blinded for submission $>$.

Our analysed sample consisted of parents and adolescents who provided any data on our primary outcome measure (the PAS), which amounted to 4,793 adults (mean age $=42.62$ years; range $=18-71 ; S D=1.75 ; 93 \%$ female) and 698 adolescents (mean age $=13.42$ years; $11-17, S D=6.25 ; 50 \%$ female $)$. The mean number of children per household was $2.01(S D=0.80)$. In total, $94 \%$ of parents/carers reported their ethnicity as white (British, Irish, other) and 59\% reported they had an undergraduate degree equivalent or above. 


\section{Measures}

Pandemic Anxiety Scale (PAS)

In order to capture the specific aspects of the pandemic which result in worry, we developed a preliminary questionnaire. First, an initial pool of items was developed by a panel of psychologists with expertise in population mental health and psychometrics. Items were designed to be short, easy-to-read, and encompass worries related to the disease itself (e.g., 'I'm worried that I will catch COVID-19') and concerns about the consequences of the pandemic and lockdown (e.g., I'm worried about the long-term impact this will have on my job prospects and the economy?). After the initial item pool was developed, members of a local community group (formed to assist vulnerable individuals during the pandemic) provided feedback on the content (relevance and breadth) and readability of the preliminary version of the scale. The version administered in the present study consisted of nine self-report items, each of which was rated on a 5point Likert scale ranging from 0 ('Strongly disagree') to 4 ('Strongly agree'). Total scores range between 0 and 36 .

\section{Psychological distress}

Common symptoms of psychological distress were assessed using two self-report measures: a subset of nine items from the Depression Anxiety Stress Scales (DASS-21) ${ }^{1}$ (Antony, Bieling, Cox, Enns, \& Swinson, 1998) in the parent sample and the Kessler Psychological Distress Scale (K6) (Kessler et al., 2002) in the adolescent sample.

\section{Demographic and health factors}

Demographic factors included respondent age and gender (male/female/other). Parents were asked to report total annual household income in five categories ranging from less than $£ 16,000$ to greater than $£ 120,000$ (see Table S2), and this information was included as an ordinal variable in our analyses. Parents were asked a series of questions about whether they, their children, or other members of the household were diagnosed with any chronic physical conditions (e.g., high blood pressure, cancer, heart disease, lung disease, other). From these questions, we derived three binary variables in the parent sample (parent condition, child condition, and other household resident condition). In the adolescent sample, we derived a similar binary variable for the study child.

\section{Analysis}

Psychometric properties of the PAS

First, we inspected the skewness and kurtosis of scores on the initial PAS items to identify, relying on conventional guidelines of values \pm 3 indicating potentially problematic items (Hair, Black, Babin, Anderson, \& Tatham, 1998). Next, we examined the latent structure of the PAS using exploratory factor analysis (EFA), which was conducted on half of the adult sample selected at random $(N=2,366)$. We then confirmed the structure using categorical confirmatory factor analysis in the second half of the adult sample $(N=2,426)$ and the full sample of adolescents $(N=697)$. Item characteristic curves (ICCs) illustrate the distribution of responses across different levels of the latent trait, and

\footnotetext{
I'Items 2, 3, 4, 6, 8, 10, 17, 18, 20. Unidimensional factor model fit: $R M S E A=0.077 ; C F I=0.973$.
} 
the precision of measurement was assessed by plotting total information functions. Finally, concurrent validity was tested by exploring correlations with the DASS and K6 scores.

\section{Demographic and health predictors of pandemic anxiety}

The associations between demographic and health predictors and pandemic anxiety were investigated using structural equation modelling (SEM). Measures of psychological distress (DASS and K6) were included as covariates in our models, to ensure that the PAS was not simply capturing general state anxiety/psychological distress. All models were estimated in Mplus version 8.3 (Muthén \& Muthén, 2017) using the weighted least squares estimator (WLSMV) estimator.

The fit of CFA and SEM models was assessed using the following indices: the chi-square statistic, the comparative fit index (CFI; Bentler, 1990), the Tucker-Lewis Index (TLI; Tucker \& Lewis, 1973), and the root mean square error of approximation (RMSEA; Steiger, 1990). CFI and TLI values of greater than 0.90 indicate acceptable model fit (Barrett, 2007). General guidelines suggest that RMSEA values of less than .05 indicate close fit and values up to .08 indicate reasonable errors of approximation ( $\mathrm{Hu} \&$ Bentler, 1999). However, research has demonstrated that the RMSEA may be impacted both by sample size and by the complexity of the model (i.e., biased in favour of models with high degrees of freedom), and thus, an upper limit of 0.10 may be more appropriate when testing simple models using smaller samples (Barrett, 2007).

\section{Results}

Descriptive statistics for all of the study variables are presented in the online supplement (Tables S1-S2).

\section{Psychometric properties of the PAS}

Inspection of the distributions of the preliminary items (Figure S1) revealed a strong ceiling effect for item 1 ('I think that COVID-19 is a very serious issue'), and this was confirmed by problematic skewness and kurtosis values (Table S3); therefore, it was removed from the scale. The remaining items all had acceptable levels of skewness and kurtosis (Hair et al., 1998).

The KMO value $(0.79)$ and Bartlett's test $\left(X^{2}(36)=9,440.65, p<.05\right)$ indicated that the data were suitable for EFA. The EFA, estimated using maximum likelihood with geomin rotation, identified a 2 -factor solution in the first random half of the adult sample. This was based on the extraction of two eigenvalues $>1$ and a parallel analysis (Figure S2). Concerns about catching and transmitting the virus loaded onto the first factor (labelled 'Disease-anxiety'), whereas the second factor tapped into worries about the consequences of the pandemic (named 'Consequence-anxiety'). The factor loadings were all in the acceptable range, with the exception of item 6 ('I'm worried we won't have enough food and other essential items') which demonstrated high cross-loadings and was removed from the final scale (Table S4). The 2-factor structure was cross-validated using CFA in the remaining adult participants and the full adolescent sample, with excellent fit and strong factor loadings (Tables S5-S6). Internal consistency (Cronbach's alpha) values for the subscales were acceptable given the low number items (range $=0.78-0.60$; 
Table S1). The correlation between the latent factors was only moderate ( 0.36 ; Table S7). Convergent validity was supported by positive and moderate correlations with the subscales of the DASS and K6 (Table S7). ICCs (Figure S3) indicated that item responses were reasonably distributed across the Likert categories, and total information functions (Figure S4) indicated that the subscales were most precise at average to moderate levels of the latent trait $( \pm 1.5 \mathrm{SD}$ s from the mean), which is a desirable feature of a population mental health measure (Ploubidis, McElroy, \& Moreira, 2019). The final 7-item version of the questionnaire accompanies this manuscript (S1) and is free to use.

\section{Demographic and health predictors of pandemic anxiety}

SEMs exploring the associations between demographic and health factors and the latent dimensions of the PAS are presented in Figures 1 and 2. Models were estimated separately for adults and adolescents, and both models demonstrated acceptable fit (RMSEA $=0.06$ /

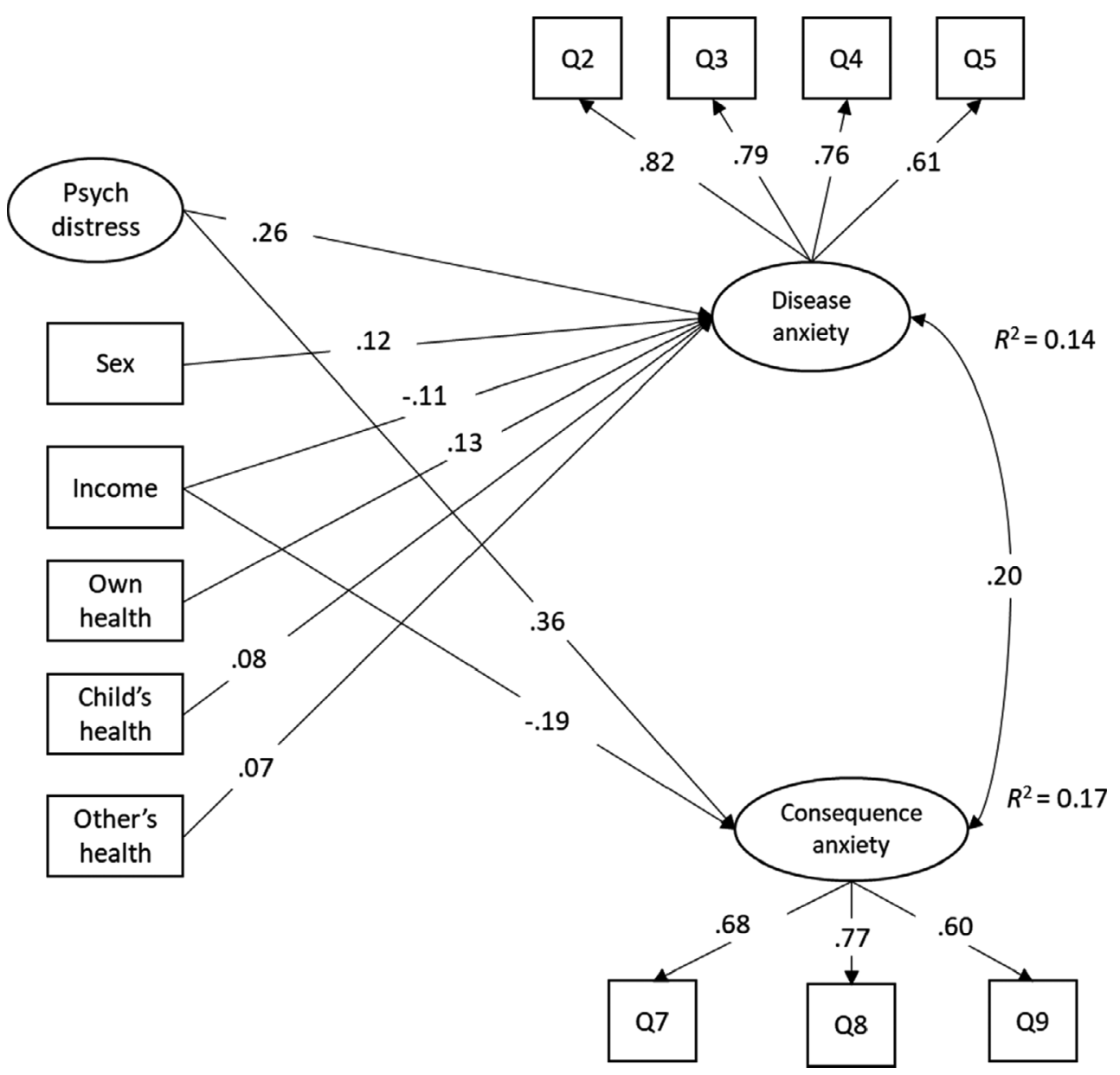

Figure I. Standardized path coefficients using the full adult sample $(N=4,320)$. Health = study parent chronic health condition. 'Male' treated as reference category for sex variable. 'No chronic condition' treated as reference category for health variables. 


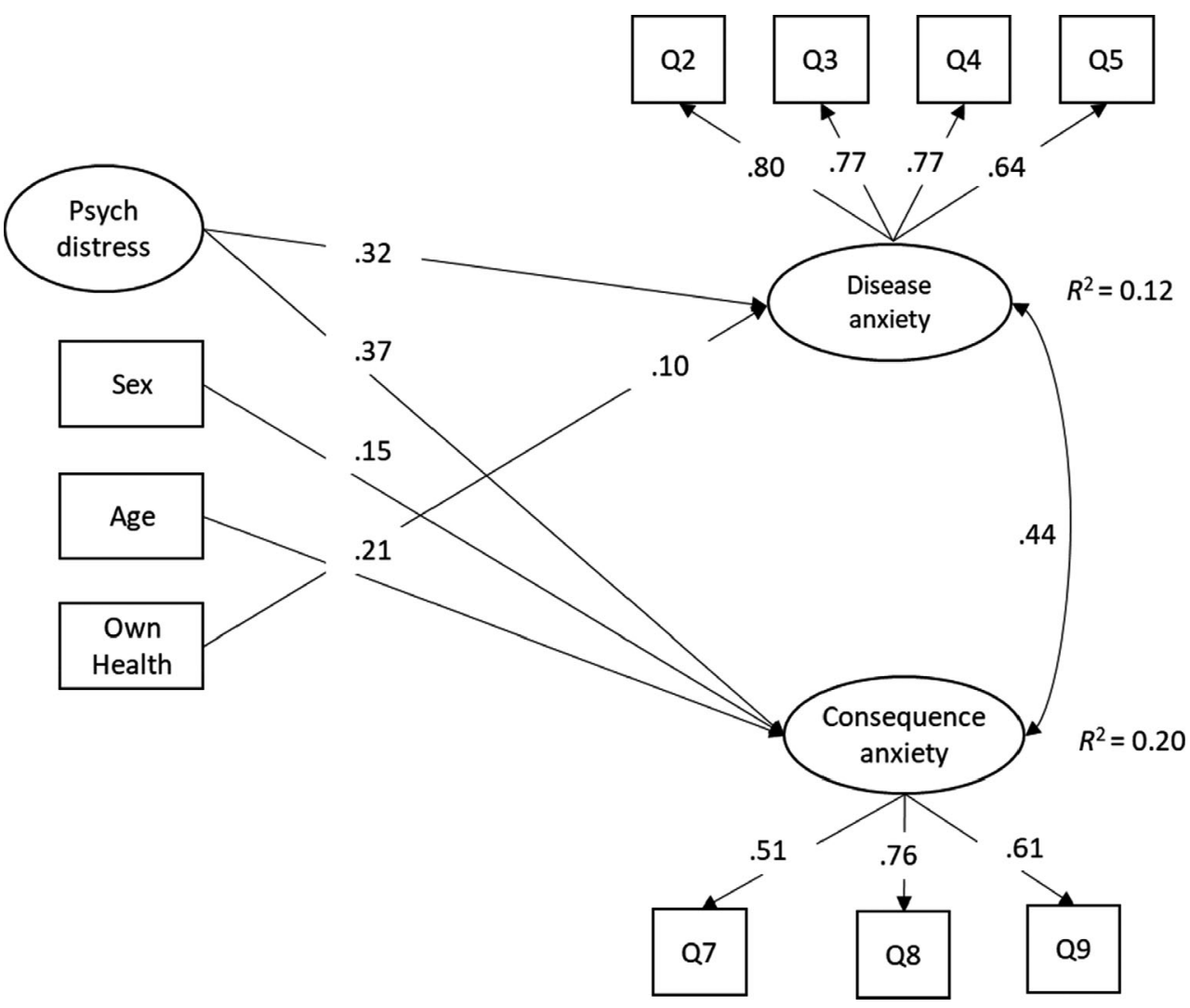

Figure 2. Standardized path coefficients using the full adolescent sample $(N=667)$. 'Male' treated as reference category for sex variable. 'No chronic condition' treated as reference category for health variable.

0.08; CFI $=0.94 / 0.92)$. In the adult sample, females scored higher on both dimensions of the PAS than males. Age was not associated with either form of pandemic anxiety in this age group. Lower household income was positively associated with both domains of the PAS. Finally, chronic physical health problems (either in relation to the reporter, their children, or other household members) were positively associated with disease-related anxiety itself.

In the adolescent sample, females and older adolescents were more likely to worry about the consequence of the pandemic, whereas those with chronic conditions were more likely to be worried about the disease itself.

\section{Discussion}

The present study aimed to profile different forms of anxiety experienced during the COVID-19 pandemic. First, in order to adequately capture such anxieties we developed a new questionnaire: the Pandemic Anxiety Scale (PAS). It is a brief 7-item measure suitable for administration in large-scale survey studies, and our initial validation work suggests strong psychometric properties in both adult and adolescent samples. This scale captures 
two forms of pandemic-related anxiety: disease anxiety and consequence anxiety. Given the modest correlation between these factors, and the specificity observed in their relations with demographic and health factors, it appears that these forms of pandemic anxiety are relatively distinct.

With regard to demographic and health factors, self-reported income was associated with both forms of anxiety, whereas physical ill-health (for both participants and other household residents) predicted disease anxiety, rather than a fear of the consequences. These patterns are likely the result of the unequal distribution of the physical and economic risks in the population (Jordan et al., 2020; McKibbin \& Fernando, 2020; Office for National Statistics, 2020). For instance, given individuals with underlying health conditions are at greater risk of hospitalization and/or death due to COVID-19, it is unsurprising that they would be more concerned about contracting the disease that those without health problems. Furthermore, people with less financial security (e.g., lower levels of income) may stand to suffer more in terms of short- and long-term economic disruption (McKibbin \& Fernando, 2020); therefore, worries about the consequences of the pandemic are understandable. Together, these findings provide initial evidence that the mental health impact of COVID-19, much like the physical health impact, may be more concentrated in certain sub-groups within the wider population (Office for National Statistics, 2020).

In addition, we found that females scored significantly higher than males on disease anxiety in the parent sample. This association could be reflective of the increased burden placed on women (e.g., caring responsibilities); however, this finding should be interpreted with some caution given the vast majority of participants were female (McLaren, Wong, Nguyen, \& Mahamadachchi, 2020). Nevertheless, in the adolescent sample, which had an even gender ratio, females scored significantly higher than males on consequence anxiety, suggesting meaningful gender difference in anxiety due to the pandemic. In this sample, age was also positively associated with consequence anxiety, which may indicate that the pandemic is a source of particular anxiety amongst older adolescents who now face considerable uncertainties in terms of their educational and economic prospects (Ahmed et al., 2020). Although age did not affect either form of anxiety in the parent sample, this is likely a result of the narrow age range of participants, with very few in the 'high-risk' age group for poor physical health outcomes (Office for National Statistics, 2020). As such, further research into the pandemic anxieties across different age groups, and other demographic factors not captured in our current data, is required.

The present study has both strengths and limitations to consider. Strengths include a large, multi-generation sample, and the use of a comprehensive range of psychometric techniques to validate our new measure. However, it must be noted that our opportunity sample of parents is predominantly female, affluent, and within a limited age range, which may impact the generalizability of our results. Despite this limitation, our findings have clear implications. First, anxieties arising due to the COVID-19 pandemic are multifaceted, and the PAS is a short, reliable, and valid measure of these concerns. Second, these anxieties are likely to be differentially associated with demographic, social, and health factors in both adolescents and adults. It is therefore important to consider such group differences when developing strategies to mitigate the mental health impact of the COVID-19 pandemic (Holmes et al., 2020).

In summary, we present the PAS, a short, reliable measure of two distinct types of anxieties arising due to the COVID-19 pandemic (disease anxiety and consequence anxiety). Our findings suggest that the anxieties arising from this unprecedented 
pandemic can vary substantially based on demographic and health characteristics. In particular, older adolescents and people with lower income appear particularly concerned about the long-term consequence of the pandemic, whereas those with underlying physical health conditions are more concerned about the disease itself. Therefore, it appears that inequalities in the mental health consequences of COVID-19 mirror those of the physical health consequences.

\section{Conflicts of interest}

All authors declare no conflict of interest.

\section{Author contribution}

Eoin McElroy (Conceptualization; Data curation; Formal analysis; Investigation; Methodology; Writing - original draft; Writing - review and editing) Praveetha Patalay (Conceptualization; Formal analysis; Investigation; Methodology; Project administration; Supervision; Writing - original draft; Writing - review and editing) Bettina Moltrecht (Conceptualization; Data curation; Investigation; Methodology; Writing - original draft; Writing - review and editing) Mark Shevlin (Conceptualization; Data curation; Formal analysis; Methodology; Supervision; Writing - review and editing) Adrienne Shum (Data curation; Investigation; Methodology; Project administration) Cathy Creswell (Conceptualization; Investigation; Methodology; Project administration; Supervision; Writing original draft; Writing - review and editing) Polly Waite (Conceptualization; Data curation; Investigation; Methodology; Project administration; Supervision; Writing original draft; Writing - review and editing).

\section{Data availability statement}

The data that support the findings of this study are available from the senior authors upon reasonable request.

\section{References}

Ahmed, F., Ahmed, N. E., Pissarides, C., \& Stiglitz, J. (2020). Why inequality could spread COVID-19. The Lancet Public Health, 5, e240. https://doi.org/10.1016/S2468-2667(20)30085-2

Ahorsu, D. K., Lin, C.-Y., Imani, V., Saffari, M., Griffiths, M. D., \& Pakpour, A. H. (2020). The fear of COVID-19 scale: Development and initial validation. International Journal of Mental Health and Addiction. https://doi.org/10.1007/s11469-020-00270-8

Antony, M. M., Bieling, P. J., Cox, B. J., Enns, M. W., \& Swinson, R. P. (1998). Psychometric properties of the 42-item and 21-item versions of the Depression Anxiety Stress Scales in clinical groups and a community sample. Psychological Assessment, 10, 176-181. https://doi.org/10.1037/10403590.10 .2 .176

Banks, J., \& Xu, X. (2020). The mental health effects of the first two months of lockdown and social distancing during the Covid-19 pandemic in the UK. London: Institute for Fiscal Studies.

Barrett, P. (2007). Structural equation modelling: Adjudging model fit. Personality and Individual Differences, 42, 815-824. https://doi.org/10.1016/j.paid.2006.09.018

Bentler, P. M. (1990). Comparative fit indexes in structural models. Psychological Bulletin, 107(2), 238-246. https://doi.org/10.1037/0033-2909.107.2.238 
Hair, J. F., Black, W. C., Babin, B. J., Anderson, R. E., \& Tatham, R. L. (1998). Multivariate data analysis, Vol. 5. Upper Saddle River, NJ: Prentice Hall.

Holmes, E. A., O'Connor, R. C., Perry, V. H., Tracey, I., Wessely, S., Arseneault, L., . . Bullmore, E. (2020). Multidisciplinary research priorities for the COVID-19 pandemic: A call for action for mental health science. The Lancet Psychiatry, 7, 547-560. https://doi.org/10.1016/S2215-0366 (20)30168-1

Hu, L. T., \& Bentler, P. M. (1999). Cutoff criteria for fit indexes in covariance structure analysis: Conventional criteria versus new alternatives. Structural Equation Modeling: A Multidisciplinary Journal, 6(1), 1-55. https://doi.org/10.1080/10705519909540118

Jordan, R. E., Adab, P., \& Cheng, K. (2020). Covid-19: Risk factors for severe disease and death. British Medical Journal, 368, m1198. https://doi.org/10.1136/bmj.m1198

Kessler, R. C., Andrews, G., Colpe, L. J., Hiripi, E., Mroczek, D. K., Normand, S.-L., . . Zaslavsky, A. M. (2002). Short screening scales to monitor population prevalences and trends in non-specific psychological distress. Psychological Medicine, 32, 959-976. https://doi.org/10.1017/ S0033291702006074

Lee, S. A. (2020). Coronavirus Anxiety Scale: A brief mental health screener for COVID-19 related anxiety. Death Studies, 44, 393-401. https://doi.org/10.1080/07481187.2020.1748481

McKibbin, W. J., \& Fernando, R. (2020). Macroeconomic impacts of COVID-19: Seven scenarios. SSRN Electronic Journal. https://doi.org/10.2139/ssrn.3547729

McLaren, H. J., Wong, K. R., Nguyen, K. N., \& Mahamadachchi, K. N. D. (2020). Covid-19 and women's triple burden: Vignettes from Sri Lanka, Malaysia, Vietnam and Australia. Social Sciences, 9, 87. https://doi.org/10.3390/socsci9050087

Muthén, L. K., \& Muthén, B. O. (2017). Mplus user's guide (8th ed.). Los Angeles, CA: Muthén \& Muthén.

Office for National Statistics. (2020). Coronavirus (COVID-19) round-up/Deaths involving COVID-19. Retrieved from https://www.ons.gov.uk/peoplepopulationandcommunity/healtha ndsocialcare/conditionsanddiseases/articles/coronaviruscovid19roundup/2020-03-26\#c oviddeaths

Ploubidis, G. B., McElroy, E., \& Moreira, H. C. (2019). A longitudinal examination of the measurement equivalence of mental health assessments in two British birth cohorts. Longitudinal and Life Course Studies, 10, 471-489. https://doi.org/10.1332/ 175795919X15683588979486

Shevlin, M., McBride, O., Murphy, J., Miller, J. G., Hartman, T. K., Levita, L., \& Stocks, T. V. (2020). Anxiety, depression, traumatic stress, and COVID-19 related anxiety in the UK general population during the COVID-19 pandemic. https://doi.org/10.31234/osf.io/hb6nq

Steiger, J. H. (1990). Structural model evaluation and modification: An interval estimation approach. Multivariate Behavioral Research, 25, 173-180. https://doi.org/10.1207/s15327906mb r2502_4

Taylor, S., Landry, C., Paluszek, M., Fergus, T. A., McKay, D., \& Asmundson, G. J. (2020). Development and initial validation of the COVID Stress Scales. Journal of Anxiety Disorders, 72 , 102232. https://doi.org/10.1016/j.janxdis.2020.102232

Tucker, L. R., \& Lewis, C. (1973). A reliability coefficient for maximum likelihood factor analysis. Psychometrika, 38(1), 1-10. https://doi.org/10.1007/BF02291170

Wang, G., Zhang, Y., Zhao, J., Zhang, J., \& Jiang, F. (2020). Mitigate the effects of home confinement on children during the COVID-19 outbreak. The Lancet, 395, 945-947. https://doi.org/10.1016/ S0140-6736(20)30547-X

Wu, P., Fang, Y., Guan, Z., Fan, B., Kong, J., Yao, Z., . . Lu, J. (2009). The psychological impact of the SARS epidemic on hospital employees in China: Exposure, risk perception, and altruistic acceptance of risk. The Canadian Journal of Psychiatry, 54,302-311. https://doi.org/10.1177/ 070674370905400504

Received I 4 May 2020; revised version received 24 July 2020 


\section{Supporting Information}

The following supporting information may be found in the online edition of the article:

Supplementary Material S1. Pandemic Anxiety Scale (PAS)

Table S1. Descriptive statistics for continuous variables

Table S2. Counts and percentages for categorical variables used in analysis

Table S3. Skewness and Kurtosis values of items based on the full sample $(N=4,793)$

Table S4. EFA Loadings from the first random half of adult sample $(N=2,366)$

Table S5. Alternative factor models tested on the second half of the adult sample $(N=2,426)$ and full adolescent sample $(N=697)$

Table S6. CFA Loadings from second random half of adult sample $(N=2,426)$ and adolescent $(N=697)$ sample

Table S7. Standardised correlation coefficients between latent PAS dimensions and established measures of psychological distress

Figure S1. Distributions of initial pool of 9 PAS items

Figure S2. Scree plot from EFA using the first random half of the adult sample $(N=2,366)$

Figure S3. Item characteristic curves for the final 7 items, based on the second randomly split adult sample $(N=2,426)$

Figure S4. Total information curves for fear (top) and consequences (bottom) subscales, based on the second randomly split adult sample $(N=2,426)$ 